\title{
Avaliação metabólica das lesões de isquemia e reperfusão cerebrais após oclusão bilateral das artérias carótidas comuns: estudo experimental em ratos ${ }^{1}$
}

\author{
Metabolic evaluation of ischemic and reperfusion brain injury following bilateral \\ occlusion of common carotid arteries: an experimental study in rats
}

\author{
Luiz Roberto Franklin Muniz², Mário Henrique Girão Faria ${ }^{3}$, \\ Paulo Roberto Leitão de Vasconcelos ${ }^{4}$
}

1. Pesquisa realizada no Laboratório de Cirurgia Experimental - LABCEX do Departamento de Cirurgia, Faculdade de Medicina - Universidade Federal do Ceará (UFC).

2. Neurocirurgião. Mestrando em Cirurgia do Departamento de Cirurgia da Faculdade de Medicina - UFC.

3. Estudante da Faculdade de Medicina - UFC.

4. Professor Doutor do Departamento de Cirurgia e Coordenador do Curso de Pós-Graduação strictu sensu em Cirurgia da Faculdade de Medicina - UFC.

\section{RESUMO}

OBJETIVO: Caracterizar as alterações no metabolismo energético após isquemia/reperfusão cerebral através de um modelo experimental de isquemia global reversível por simples oclusão das artérias carótidas comuns (ACCs) em ratos da linhagem Wistar.

MÉTODOS: Quarenta e oito ratos Wistar machos foram distribuídos aleatoriamente em dois grupos (C Controle; T-Teste) e cada um deles redistribuídos em quatro tempos de estudos. Após oclusão bilateral das ACCs por 30min, permitiu-se reperfusão aos animais do grupo T nos tempos 0, 5, 10 e 15min. Foram coletadas amostras de tecido cerebral e sangue arterial sistêmico e quantificados os metabólitos D-glicose (GLI), piruvato (PRV), lactato (LCT) e adenosina trifosfato (ATP).

RESULTADOS: Observou-se aumento nos níveis sangüíneos de GLI e PRV de, respectivamente, 85,00\% $(p<0,05)$ e 51,72\% ( $<<0,01)$ aos 5 min de reperfusão, refletindo uma resposta sistêmica à lesão isquêmica cerebral. O LCT cerebral manteve-se estável, apesar da redução de 52,66\% (p<0,05) na concentração sanguínea aos $15 \mathrm{~min}$ de reperfusão. As concentrações cerebrais de ATP caíram 85,40\% $(\mathrm{p}<0,05)$ após isquemia, seguindo-se aumento de 3.033,40\% (p<0,05) aos 5min de reperfusão, evidenciando a retomada da respiração celular via cadeia fosforilativa.

CONCLUSÕES: O modelo experimental proposto produziu isquemia global transitória parcial, com repercussão sistêmica. As maiores alterações metabólicas foram verificadas após os primeiros minutos de reperfusão, tipificando a lesão de reperfusão. Tais constatações mostram sua utilidade como ferramenta no estudo da fisiopatologia e de recursos terapêuticos da doença cerebrovascular isquêmica.

DESCRITORES: Isquemia cerebral. Reperfusão. Metabolismo. Ratos.

\section{ABSTRACT}

PURPOSE: To describe alterations on the energy metabolism after cerebral ischemia/reperfusion, through an experimental model of reversible global ischemia, by simple occlusion of common carotid arteries (CCAs) in rats of Wistar lineage. 
METHODS: Forty-eight male Wistar rats were randomly distributed on two groups (C - Control; $\mathrm{T}$-Test) and further redistributed into four times sets of study. After bilateral occlusion of CCAs for 30min, the animals of group T were allowed reperfusion for 0, 5, 10 and $15 \mathrm{~min}$. Samples of cerebral tissue and systemic arterial blood were collected and the metabolites D-glucose (GLI), pyruvate (PRV), lactate (LCT) and adenosine triphosphate (ATP) quantified.

RESULTS: Increases of GLI and PRV blood values of, respectively, 85,00\% $(p<0,05)$ and $51,72 \%(p<0,01)$ were observed at $5 \mathrm{~min}$ of reperfusion, which reflect a systemic response to the cerebral ischemia. The brain's LCT remained stable despite a reduction of $52,66 \%(p<0,05)$ in its blood concentration at $15 \mathrm{~min}$ of reperfusion. The cerebral concentrations of ATP decreased $85,40 \%(p<0,05)$ after ischemia, followed by an augmentation of 3.033,40\% $(\mathrm{p}<0,05)$ at $5 \mathrm{~min}$ of reperfusion, becoming evident the recovery of cell respiration by fosforilation pathway.

CONCLUSIONS: The proposed experimental model produced partial transitory global ischemia, with systemic repercussions. The greatest metabolic alterations were verified in the initial minutes of reperfusion, typifying reperfusion injury. Such observations prove its value as an important instrument in the study of physiopathology and therapeutic sources of ischemic cerebrovascular disease.

KEY WORDS: Brain ischemia. Reperfusion. Metabolism. Rats.

\section{Introdução}

As doenças vasculares cerebrais constituem atualmente a terceira causa de morte, sendo ainda responsáveis por consideráveis índices de morbidade. ${ }^{1,2}$ Dentre estas, 85 a 90\% ocorrem por mecanismo isquêmico. ${ }^{3}$

No Brasil, para o ano de 2004, estão previstos cerca de 21.000 novos casos de "acidentes" vasculares cerebrais (AVCs) isquêmicos, calculandose os gastos diretos, somente com internações, em torno de 13 milhões de reais. ${ }^{4}$ Nos EUA, 600.000 pessoas são atendidas por ano vítimas de episódio novo ou recorrente de AVC isquêmico, com custo médio anual de 43,3 bilhões de dólares. ${ }^{5}$

Dessa forma, percebe-se a importância do estudo sistemático da fisiopatologia dos mecanismos envolvidos na lesão neuronal isquêmica e de seu tratamento com o objetivo de formular estratégias que reduzam tanto a mortalidade como as diversas seqüelas neurológicas advindas de tal patologia.

Neste contexto, os modelos experimentais para estudo dos eventos isquêmicos e de reperfusão cerebral in vivo mostram-se fundamentais. Para tanto, mamíferos roedores como ratos constituem promissor recurso experimental por apresentarem semelhanças anátomo-fisiológicas com a espécie humana e relativa homogeneidade interespécie. ${ }^{6}$

Variados modelos de isquemia/reperfusão cerebral através da ligadura bilateral das artérias carótidas comuns (ACCs) utilizando ratos já foram propostos. Todavia, os mesmos relatam insucesso na obtenção do evento isquêmico, justificado pela rica e extensa circulação colateral evidenciada nestes animais através do polígono de Willis, das anastomoses término-terminais e dos ramos corticais provindos do sistema carotídeo interno e externo. ${ }^{7,8,}$ ${ }^{9}$ Baseando-se nestes argumentos, manobras sofisticadas de hipotensão arterial sistêmica, elevação da pressão liquórica, seleção genética de ratos hipertensos e exposição a ambiente hipóxico têm sido impostas para a efetivação do evento isquêmico, dificultando e elevando os custos dos experimentos. ${ }^{6}$

O presente estudo objetivou caracterizar as alterações metabólicas seguintes à isquemia/ reperfusão cerebral através de um modelo experimental de isquemia global reversível do prosencéfalo por oclusão de dois vasos (ACCs), sem manobras ou recursos acessórios, em ratos da linhagem Wistar.

\section{Métodos}

O estudo foi realizado de acordo com as Normas Internacionais para a Pesquisa Biomédica em Animais (1990) e de acordo com a Lei Federal $n^{\circ}$. 6.638, de 08 de maio de $1979 .{ }^{10}$ Foram utilizados 48 ratos machos da linhagem Wistar (Ratus norvegicus, Rodentia Mammalia) procedentes do Biotério Central da Universidade Federal do Ceará, com peso 
corporal médio de 406,23 $\pm 52,21 \mathrm{~g}$. Estes animais permaneceram abrigados a $24^{\circ} \mathrm{C}$ em ciclos luz/escuro de 12 horas, sendo observados quanto às condições gerais de saúde durante vinte dias, recebendo ração padrão e água ad libitum no Biotério do Laboratório de Cirurgia Experimental - LABCEX.

Os animais foram aleatória e eqüitativamente distribuídos em dois grupos: Grupo C (controle) e Grupo T (teste). Cada grupo foi redistribuído em 04 tempos de estudos. Os animais do Grupo T foram submetidos às manobras cirúrgicas com oclusão das ACCs por 30 minutos e estudados no tempo final do processo isquêmico ou $0 \mathrm{~min}$ de reperfusão, e aos 5 , 10 e 15 minutos de reperfusão. Já os animais do Grupo $\mathrm{C}$ foram submetidos a todos os procedimentos cirúrgicos, exceto a oclusão bilateral das ACCs, e estudados nos mesmos tempos do Grupo T.

Os ratos foram anestesiados através de uma associação de cloridrato de cetamina $(90 \mathrm{mg} / \mathrm{kg}$ ) e cloridrato de xilasina $(10 \mathrm{mg} / \mathrm{kg})$ administrada simultaneamente por via intraperitoneal. Após anestesia, procedeu-se cervicotomia mediana longitudinal anterior com individualização e reparo bilateral das ACCs a $0,5 \mathrm{~cm}$ de sua bifurcação em artérias carótidas externa e interna (ponto de oclusão). De acordo com as manobras cirúrgico-experimentais previstas para cada grupo, realizou-se oclusão bilateral e concomitante das ACCs com clipes vasculares tipo bulldog (Figura 1). Na seqüência, realizou-se laparotomia mediana longitudinal anterior, dissecando e reparando a artéria aorta abdominal abaixo das artérias renais e acima das artérias ilíacas comuns, objetivando posterior punção arterial. Seguiu-se craniectomia, utilizando-se rebolo flexível dupla face acoplada a um micromotor elétrico a 20.000rpm para osteotomia, procedendo-se posterior abertura dural para acesso aos hemisférios cerebrais (Figura 2).

Realizadas todas as manobras cirúrgicas planejadas e passados os tempos estabelecidos, foram retirados o hemisfério cerebral esquerdo (aleatoriamente definido) e amostras de sangue da artéria aorta abdominal de cada animal.

O hemisfério cerebral foi imediatamente congelado em nitrogênio líquido e pulverizado com uso de gral e pistilo. O material obtido, assim como as amostras sangüíneas, foi acondicionado em ácido perclórico a $10 \%$, estocado a $-4^{\circ} \mathrm{C}$ e destinado aos estudos enzimáticos: dosagens de D-glicose (GLI),

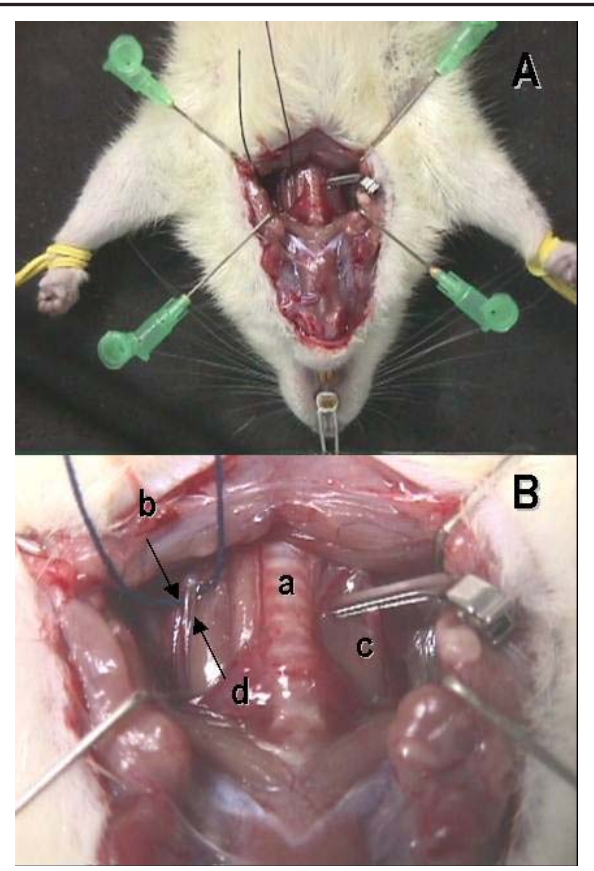

FIGURA 1 - Cervicotomia mediana longitudinal anterior. (A) Visão geral; (B) Detalhe: (a) traquéia, (b) artéria carótida comum esquerda reparada com fio cirúrgico, (c) artéria carótida comum direita ocluída por clipe vascular e (d) nervo vago.

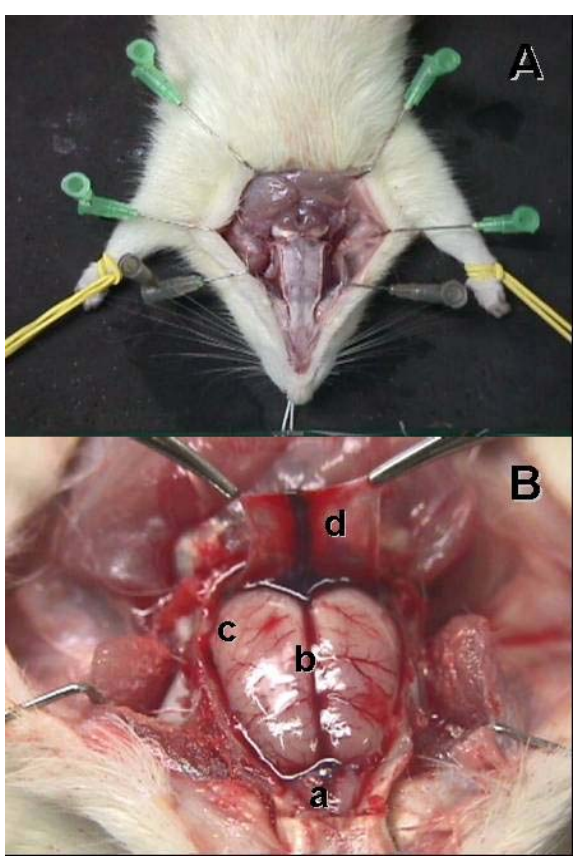

FIGURA 2 - Craniectomia. (A) Exposição do crânio; (B) Secção da dura-máter e exposição do encéfalo; (a) lobo olfatório, (b) fissura mediana, (c) hemisfério cerebral e (d) dura-máter.

piruvato (PRV), lactato (LCT) e adenosina trifosfato (ATP). As concentrações destes metabólitos, in vivo, foram calculadas em ìmol/g de tecido e em ìmol/mL de sangue. ${ }^{11}$ 
Os resultados foram expressos como Média \pm Desvio Padrão da Média, sendo utilizados os testes U de Mann-Whitney e t de Student para as análises estatísticas. ${ }^{12}$ Foram considerados estatisticamente significantes os valores com $\mathrm{p}<0,05$.

\section{Resultados}

Observou-se significante aumento dos níveis glicêmicos no Grupo T após $5 \mathrm{~min}$ de reperfusão, quando comparado ao tempo 0min (85,0\%). Já no tecido cerebral, as concentrações de glicose no Grupo T apresentaram uma elevação de aproximadamente 41,5\%, tanto em relação aos tempos anteriores quanto ao Grupo C, aos 15min de reperfusão (Figura 3).

O mesmo comportamento ocorreu com o piruvato, que demonstrou significante elevação de 51,72\% em suas concentrações sangüíneas, após 5 min de reperfusão, em relação ao Grupo anterior (Grupo T no tempo 0min) e de 29,02\% em relação ao grupo controle (Grupo C no tempo $5 \mathrm{~min}$ ), seguindo-se de queda significante de suas concentrações sistêmicas (30,44\%) aos níveis iniciais (tempo $0 \mathrm{~min}$ ) logo aos $10 \mathrm{~min}$ de reperfusão. Contudo, o piruvato apresentou significante aumento de suas concentrações a nível cerebral no grupo T já aos $5 \mathrm{~min}$ de reperfusão, permanecendo elevadas. Aos 15min houve decréscimo de significância estatística em relação às concentrações determinadas nos tempos anteriores (5 e 10min). Observa-se ainda significante elevação dos níveis cerebrais desse metabólito, no grupo controle, aos 10 minutos (Figura 4).

Quanto à lactacemia, percebeu-se queda significante (52,66\%) em sua concentração no tempo 15min no Grupo T em relação ao mesmo grupo no tempo anterior. Não foram observadas diferenças significantes nas concentrações cerebrais de lactato nos diferentes grupos estudados (Figura 5).

Houve decréscimo de $85,11 \%$ nas concentrações de adenosina trifosfato cerebral no Grupo T em relação ao C no tempo 0min. Expressivo aumento de $1.660,78 \%$ e de $3.033,40 \%$ ocorreu nas concentrações desse metabólito aos $5 \mathrm{~min}$ de reperfusão, quando analisado comparativamente ao grupo teste no tempo 0min e ao controle no tempo $5 \mathrm{~min}$, respectivamente. Seguiu-se redução em semelhante intensidade, logo aos $10 \mathrm{~min}$, para os níveis

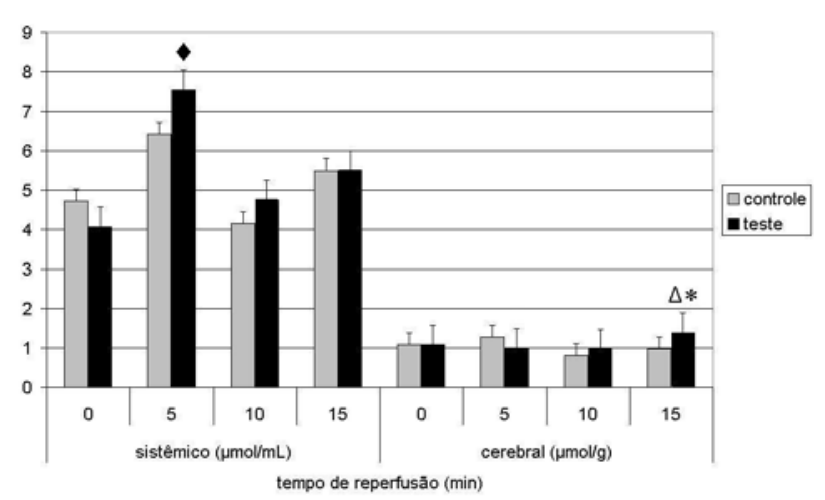

$\Delta \mathrm{p}<0,05$ em relação ao grupo controle (teste U de Mann-Whitney) - $\mathrm{p}<0,05$ em relação ao grupo anterior (teste t de Student) * $\quad \mathrm{p}<0,01$ em relação ao grupo anterior (teste t de Student)

FIGURA 3 - Concentrações sangüíneas e cerebrais de glicose mensuradas nos diferentes tempos de reperfusão entre os grupos controle e teste.

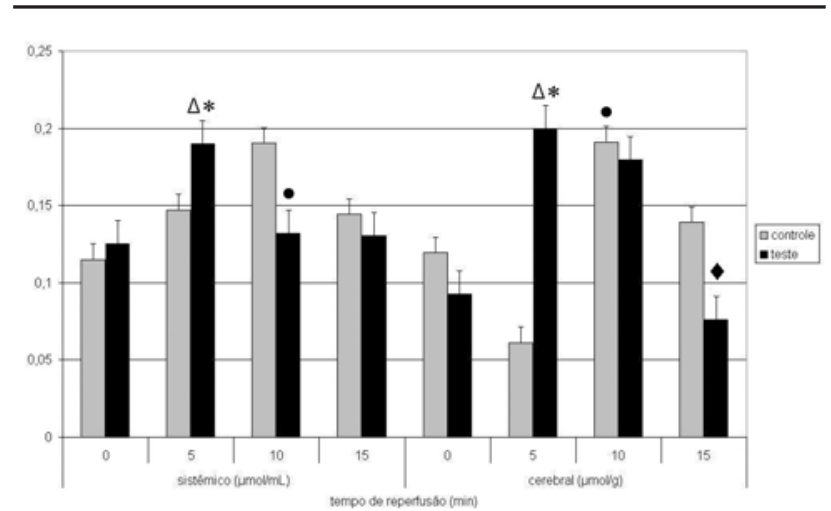

$\Delta \mathrm{p}<0,05$ em relação ao grupo controle (teste $U$ de Mann-Whitney)

- $\mathrm{p}<0,05$ em relação ao grupo anterior (teste $t$ de Student)

* $\mathrm{p}<0,01$ em relação ao grupo anterior (teste t de Student)

- $\mathrm{p}<0,005$ em relação ao grupo anterior (teste t de Student)

FIGURA 4 - Concentrações sangüíneas e cerebrais de piruvato mensuradas nos diferentes tempos de reperfusão entre os grupos controle e teste.

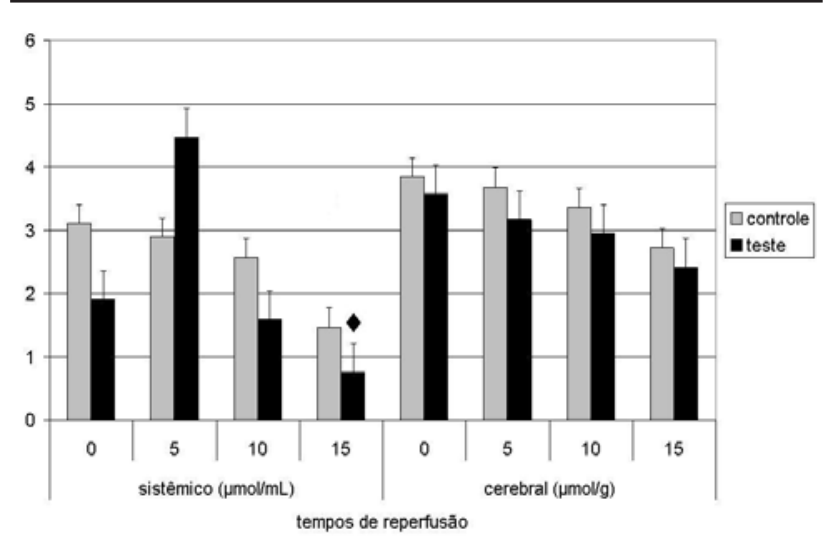

- $\mathrm{p}<0,05$ em relação ao grupo anterior (teste t de Student)

FIGURA 5 - Concentrações sistêmicas e cerebrais de lactato mensuradas nos diferentes tempos de reperfusão entre os grupos controle e teste. 
do controle. Neste último tempo, as concentrações de ATP no grupo controle mostraram elevação significante em relação ao tempo anterior (5min) (Figura 6).

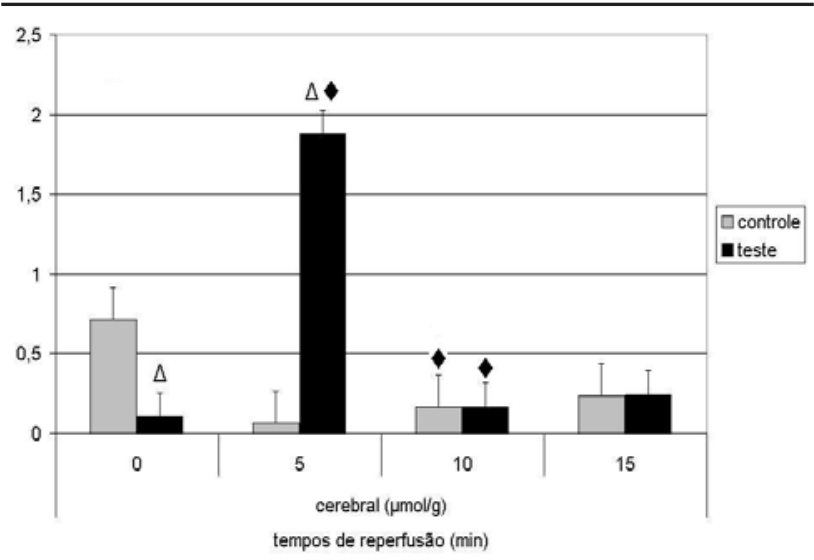

$\Delta \mathrm{p}<0,05$ em relação ao grupo controle (teste U de Mann-Whitney) - $\mathrm{p}<0,05$ em relação ao grupo anterior (teste t de Student)

FIGURA 6 - Concentrações cerebrais de ATP mensuradas nos diferentes tempos de reperfusão entre os grupos controle e teste.

\section{Discussão}

O significante aumento da glicemia após $5 \mathrm{~min}$ de reperfusão no Grupo T apresenta-se como uma resposta conjuntural à lesão cerebral isquêmica recente, desencadeando uma maior oferta compensatória desse metabólito, principal combustível energético neuronal, possivelmente através da disponibilização de suas reservas hepáticas e musculares pela via da glicogenólise., ${ }^{2,5}$

Nesse contexto de maior oferta energética observa-se, no mesmo tempo, elevação sangüínea do piruvato, produto oriundo da glicólise. O aumento concomitante das concentrações cerebrais de piruvato demonstra a avidez do encéfalo pósisquêmico em realizar a glicólise utilizando-se da suplementação de glicose sangüínea, ofertada ao cérebro após a reperfusão. ${ }^{6,13}$

Percebe-se redução da lactacemia nos animais do Grupo T, atingindo concentrações inferiores àquelas verificadas no Grupo $\mathrm{C}$, no tempo $15 \mathrm{~min}$. Tal comportamento indica redução da oferta de lactato com o decorrer da reperfusão, representando a provável retomada do metabolismo aeróbico em tecidos periféricos. ${ }^{13,14}$ Já no tecido cerebral, a lesão isquêmica não induziu alterações nas concentrações de lactato.
Há significante queda das concentrações cerebrais de ATP após 30 minutos de oclusão das artérias carótidas comuns (0min de reperfusão), comprovando o sucesso na indução da isquemia cerebral pelo modelo proposto. O significante incremento das concentrações de ATP no tecido cerebral aos $5 \mathrm{~min}$ de reperfusão reflete proporcional aumento da respiração celular via cadeia fosforilativa, decorrente da restauração da circulação sangüínea, caracterizando a lesão de reperfusão. Aos 10 minutos de reperfusão, houve redução das concentrações de ATP no tecido cerebral para níveis semelhantes aos aferidos nos animais do grupo controle. Isto sugere uma adaptação à lesão de reperfusão em tempos "tardios” pós-isquemia., 13

Após intensa atividade glicolítica, observa-se tendência ao restabelecimento do perfil energético cerebral aos 15 minutos de reperfusão. Nota-se diminuição da glicólise pela queda nos níveis cerebrais de piruvato, conseqüente aumento nas dosagens cerebrais de glicose e redução de ATP aos níveis do grupo controle. Além de reafirmar a mobilização sistêmica da glicose para o tecido cerebral recéminjuriado, demonstra-se o caráter reversível do processo isquêmico desencadeado, com propensão à homeostase..$^{13,14}$

De um modo geral, confirma-se o estabelecimento de isquemia cerebral, ${ }^{15}$ embora sem evidente anaerobiose, com alterações metabólicas desencadeadas principalmente durante a reperfusão sanguínea cerebral, caracterizando o chamado “complexo de lesão de reperfusão”: alterações funcionais metabólicas e redução local da perfusão pelos fenômenos de "não-refluxo". 5,6

\section{Conclusões}

-A oclusão bilateral das artérias carótidas comuns em ratos Wistar produziu isquemia global transitória parcial, comprovada pela redução de ATP no tecido cerebral, com repercussão sistêmica;

- As alterações metabólicas mais significativas foram verificadas nos primeiros minutos de reperfusão, caracterizadas pela mobilização e consumo de substratos energéticos sistêmicos e conseqüente superprodução de ATP, tipificando a "lesão de reperfusão";

- O modelo experimental mostrou-se simples e reprodutível, podendo ser utilizado como ferramenta 
para estudos sobre a fisiopatologia da isquemia e reperfusão cerebrais, bem como para o desenvolvimento de propostas e recursos terapêuticos para tais lesões.

\section{Referências}

1. Adams RD, Victor M, Rooper AH. Adam's \& Victor's Principles of Neurology. 6ed. Washington: Hardcover; 1998.

2. Hagberg H, Ichord R, Palmer C, Yager JY, Vannucci SJ. Animal models of developmental brain injury: relevance to humam disease. A summary of the panel discussion from the Third Hershey Conference on Developmental Cerebral Blood Flow and Metabolism. Dev Neurosci 2002;24(5):364-6.

3. Zauner A, Daugherty WP, Bullock MR, Wamer DS. Brain oxygenation and energy metabolism. Part I: biological function and pathophysiology. Neurosurgery 2002;51(2):289-301.

4. DATASUS. Sistema de Informação Hospitalar (SIH). Sistema Único de Saúde. Ministério da Saúde. Governo do Brasil; 2003.

5. White BC, Sullivan JM, DeGracia DJ, O’Neil BJ, Neumar RW, Grossman RW, Rafols JA, Krause GS. Brain ischemia and reperfusion: molecular mechanisms of neuronal injury. J Neurol Sci 2000;179:1-33.

6. Castro e Silva Jr O, Zucoloto S, Beer Jr A. Modelos Experimentais de Pesquisa em Cirurgia. São Paulo: Robe editorial; 1998.
7. Eklöf B, Siesjö BK. The effect of bilateral carotid artery ligation upon the blood flow and the energy state of the rat brain. Acta Physiol Scand 1972;86:155-65.

8. Gisberg MD, Busto R. Rodent models of cerebral ischemia. Stroke 1989;20:1627-42.

9. Toda S; Ikeda Y, Teramoto A, Hirakawa K, Uekusa K. Highly reproducible rat model of reversible forebrain ischemia - modified four-vessel occlusion model and its metabolic features. Acta Neurochir 2002;144(12):1297-304.

10.Brasil. Lei Federal $n^{\circ}$. 6.638, de 8 de maio de 1979. Estabelece normas para a prática didático-científica da vivissecção de animais e determina outras providências. In: Brasil. Coleção de leis de 1979; atos legislativos do poder executivo. Leis de abril a junho. Brasília, Departamento de Imprensa Nacional, 1979:33-4.

11.Hohorst apud Vasconcelos PRL. Hepatic metabolism during sepsis [Tese de Doutorado]. Oxford University; 1987.

12.Fisher RA, Yates F. Statistical tables for biological, agricultural and medical research. $5^{\mathrm{a}}$ ed. Edinburgh: Oliver and Boyd Ltd.; 1980.

13.Schurr A. Lactate, glucose and energy metabolism in the ischemic brain. Int J Mol Med 2002;10(2):131-6.

14.Phillis JW, O’Regan MH. Energy utilization in the ischemic/reperfused brain. Int Rev Neubiol 2002;51:377414.

15. Vale OC. Avaliação eletrofisiológica da ação dos antioxidantes glutation e ácido a-lipóico em um modelo animal de isquemia cerebral. Arq Neuro-Psiquiatr 2002;60(2B):510.

\section{Correspondência:}

Luiz Roberto Franklin Muniz

Faculdade de Medicina - Universidade Federal do Ceará

Departamento de Cirurgia

Rua Professor Costa Mendes, 1608/3 andar

60430-140 Fortaleza-CE

Tel.: (85)288-8063

Fax: (85)288-8064

mcirur@npd.ufc.br

Recebimento: 14/06/2004

Revisão: 21/07/2004

Aprovação: 01/09/2004

Conflito de interesse: nenhum

Fonte de financiamento: nenhuma

\section{Como citar este artigo:}

Muniz LRF, Faria MHG, Vasconcelos PRL. Avaliação metabólica das lesões de isquemia e reperfusão cerebrais após oclusão bilateral das artérias carótidas comuns: estudo experimental em ratos. Acta Cir Bras [serial online] 2004 SetOut;19(5). Disponível em URL: http://www.scielo.br/acb [também em CD-ROM].

*Figuras coloridas disponíveis em www.scielo.br/acb 